\title{
Clinical Staging and Genetic Profiling of Korean Patients with Primary Lymphedema Using Whole- Exome Sequencing: Preliminary Results
}

\section{Soo Hyun Seo}

Seoul National University Bundang Hospital, Seoul National University College of Medicine

\section{Seung Joon Lee}

Seoul National University Bundang Hospital, Seoul National University College of Medicine Joseph Kyu-hyung Park

Seoul National University Bundang Hospital, Seoul National University College of Medicine

\section{Eun Joo Yang}

Seoul National University Bundang Hospital, Seoul National University College of Medicine

\section{Boram Kim}

Seoul National University Hospital, Seoul National University College of Medicine

Jee-Soo Lee

Seoul National University Hospital, Seoul National University College of Medicine

\section{Man Jin Kim}

Seoul National University Hospital, Seoul National University College of Medicine

\section{Sung Sup Park}

Seoul National University Hospital, Seoul National University College of Medicine

\section{Moon-Woo Seong}

Seoul National University Hospital, Seoul National University College of Medicine

\section{Yujin Myung ( $\nabla$ surgene@snu.ac.kr)}

Seoul National University Bundang Hospital, Seoul National University College of Medicine

\section{Research Article}

\section{Keywords:}

Posted Date: February 2nd, 2022

DOI: https://doi.org/10.21203/rs.3.rs-1299738/v1

License: (c) (i) This work is licensed under a Creative Commons Attribution 4.0 International License. Read Full License 


\section{Abstract}

Lymphedema is a progressive disease caused by lymphatic flow blockage in the lymphatic pathway. Primary (hereditary) lymphedema is caused by genetic mutations without secondary causes. However, the underlying cause of primary lymphedema remains obscure, and gene sequencing studies on a sizable patient cohort are lacking. We performed clinical profiling on Korean primary lymphedema patients based on their phenotypes and using lymphoscintigraphy and made genetic diagnoses using a next-generation sequencing panel consisting of 60 lymphedema-related genes. Of 27 patients, $21 \%$ had lymphedema of the upper extremities, $81 \%$ had lymphedema of the lower extremities, and two patients had lymphedema in both. Based on International Society of Lymphology staging, 14, 10, and 3 patients had stage 3, 2, and 1 lymphedema, respectively. Genetic abnormalities were identified in six patients: a pathogenic variant of CELSR1 in two patients, variants of uncertain significance (VUSs) of CELSR1 in two patients, a VUS of SOS1 in one patient, and a pathogenic variant of MPI and VUSs of TIE1, FLT4, and PIEZO1 in one patient. The clinical and genetic profile of hereditary lymphedema reported here is the first such data series reported in South Korea. Our study highlights the need for genetic screening in primary lymphedema diagnosis.

\section{Introduction}

Lymphedema is a disorder of lymphatic transport caused by blockage and loss of function of the lymphatics. It is largely divided into primary lymphedema with no specific or unknown cause and secondary lymphedema with causes, such as cancer-related treatment or filiarisis. Primary lymphedema is divided into types I and II according to the age of onset of symptoms [1]. Compared with that of secondary lymphedema, the prevalence of primary lymphedema is unknown. Although previous studies report approximately $1: 100,000-500,000[2,3]$, the actual prevalence globally is estimated to be greater [4]. Primary lymphedema patients are often underdiagnosed as they do not receive an accurate diagnosis of the actual cause of edema and are subjected to various alternative tests and treatments owing to lack of accurate information about primary lymphedema [5].

Type I (congenital) and type II (pubertal onset) primary lymphedema were first described by Milroy [2, 3] and Meige [4] in 1892 and 1898, respectively. However, even decades later, the underlying cause of primary lymphedema remains obscure, except for some associated autosomal dominant VEGFR3 mutations [6, 7]. Although there have been sporadic reports of lymphatic dysfunction associated with congenital syndromes, such as lymphedema-distichiasis syndrome (associated with FOXC2) [8] and Emberger syndrome (associated with GATA2) [9], majority are case reports. Studies analyzing genetic alteration and phenotypic classification in a sizable patient cohort are lacking.

Therefore, in the present study, we aimed to perform accurate clinical profiling of Korean patients with primary lymphedema and analyze their genetic variance using whole-exome sequencing (WES).

\section{Results}




\section{Clinical characteristics of patients}

Of the 27 patients included in the study, 18 were female and 9 were male. Their mean age was 35 years (range, 1 to 77 years), whereas their average body mass index was 24.8. Notable past medical histories of patients included ongoing lung cancer $(n=1)$, hypertension $(n=4)$, and diabetes mellitus $(n=2)$. Three patients had familial history of primary lymphedema in second-degree relatives. In one of these cases, both the proband and her mother harbored a pathological variant of CELSR1 (see Case presentation).

In $41 \%$ of the patients $(n=11)$, symptoms first appeared between the ages of 20 and 40 years. In contrast, the rate of onset of symptoms before age 10 was $15 \%(n=4)$, that between the ages of 10 and 20 years was $15 \%(n=4)$, and that after age 40 was $26 \%(n=7)$. The most common lymphedema location was unilateral lower extremity $(n=18,67 \%)$, followed by bilateral lower extremity $(n=4,15 \%)$, unilateral upper extremity $(n=3,11 \%)$, bilateral upper extremity $(n=2,7 \%)$, and the face $(n=1,4 \%)$.

Based on the International Society of Lymphology (ISL) stages, stage 3 lymphedema was the most common $(n=14,52 \%)$, followed by stage $2(n=10,37 \%)$ and stage $1(n=3,11 \%)$. The average difference in volume between the involved limb and the normal limb was $16 \%$. On lymphoscintigraphy images, apparent dermal backflow with or without axillary/inguinal lymph node uptake was observed in all patients, and in the case of lymphoscintigraphy grade, most patients $(n=19)$ showed total lymphatic obstruction (stage 4-6) in the involved limb. Indocyanine-green (ICG) lymphography was also performed in all patients, and with respect to dermal backflow stages $(0-V)$, most patients showed a stardust/diffuse pattern in majority of the involved limbs at stages IV $(n=15)$ and $V(n=7)$ (Fig. 1, Table 1).

Four of the patients underwent lymphovenous anastomosis (LVA) at Hereditary Lymphedema Clinic, Seoul National University Bundang Hospital (Seongnam, South Korea), and one of these patients underwent simultaneous liposuction. Regarding history of previous surgery at other hospitals, liposuction $(n=2)$, LVA $(n=1)$, vascularized lymph node transfer $(n=1)$, and stem cell therapy $(n=1)$ were recorded.

\section{Case presentation}

A 38-year-old female presented with stage II lymphedema on her bilateral hand and stage III lymphedema on her bilateral leg, below the knee (Case 1 in Table 2). The age of onset for edema was age 11; however, the patient did not receive proper diagnosis or treatment until age 17, when she first developed cellulitis on her right foot and was subsequently hospitalized.

After primary lymphedema was diagnosed through radioisotope lymphoscintigraphy and clinical presentation, the patient developed similar symptoms in both upper extremities and continued rehabilitation treatment, including complex decongestive therapy and manual lymphatic drainage for both arms and legs. In the case of the lower extremities, there was no further change from stage III lymphedema, and the appearance was stationary. However, in the case of the upper extremities, lymphedema worsened from stage I to II despite recent rehabilitation treatment. The patient thus took 
leave of absence from her job and focused on her treatment. She then visited our clinic for LVA of both upper extremities.

Lymphoscintigraphy showed that the uptake in the left axillary lymph node was decreased, whereas the uptake in the left antecubital lymph node was increased; mild dermal backflow was observed in both forearms (Fig. 2). In the ICG examination, dermal backflow and lymphatic retention were observed in both forearms; however, the left side showed a more severe pattern than the right side (Fig. 3). Additionally, the patient's mother had similar but milder lower leg primary lymphedema and also underwent genetic testing. Similar symptoms were not confirmed in other family members, and the patient's father was not tested.

The patient then underwent LVA at two sites on each arm. Before surgery, the location of anastomosis was selected using ICG lymphangiography and high frequency ultrasonography (Fig. 4.). The patient underwent two successful LVAs on each side through a 2-cm incision and was discharged the next day without any complications from surgery. Approximately 10 months after the surgery, the patient's upper extremity lymphedema reduced to stage $\mathrm{I}$, and the edema almost disappeared. She also eventually returned to work.

\section{Germline variants of lymphedema-related genes}

The average coverage depth in the target regions of the WES panel was $160.7 \mathrm{X}$. On average, $92.8 \%$ of the bases had coverage $\geq 10 \times$ (45.2-99.5\%). Among the 27 patients, eight variants of interest were detected in eight patients: two likely pathogenic variants and six variants of uncertain significance (VUSs) (Table 1).

A nonsense variant of CELSR1, c.8446C>T (p.GIn2816*), and a frameshift variant, c.8871_8872del (p.Cys2957*), were detected in the patient presented as case 1 (Table 2). c.8446C $>T$, which was classified as a likely pathogenic variant, was also detected in the patient's symptomatic mother who was included in the study group. c.8871_8872del, which results in a premature termination codon in the penultimate exon, was classified as a VUS. This variant is expected to result in the loss of less than $2 \%$ of the protein, and the role of the truncated region is unknown. The patient's mother did not harbor this variant; however, since her asymptomatic father was not tested, it could not be confirmed whether he harbored the variant as well.

Another pathogenic variant, MPIc.13C>T (p.Arg5*), was detected in case 4 (Table 1). The MPIgene is associated with congenital disorder of glycosylation, type $\mathrm{lb}$, which is inherited in an autosomal recessive pattern [10]. However, the patient carrying this variant did not have any other symptoms related to this diagnosis. Hence, this variant could not be confirmed as a genetic cause for her lymphedema. She also had three VUSs in other genes (TIE1, FLT4, and PIEZO1), which can possibly be reclassified as variants of interest given additional evidence.

\section{Discussion}


In the present study, we analyzed the clinical characteristics and genetic profiles of 27 patients with primary lymphedema. To the best of our knowledge, this is the first clinical and genetic profiling study on a primary lymphedema cohort published from a single institution in East Asia.

To date, studies on primary lymphedema have mainly focused on reviewing clinical features [11] of patients and discussing relevant medical and surgical treatments. Since the feature of this disease was first described by Milroy and Meige, sporadic presentations of different phenotypical patterns have been reported [6,12-16], and accordingly, the disease pattern and diagnostic criteria have changed over the years $[1,3,11,17,18]$. Regarding causative mutations, specific genes have not been reported; however, gene mutations have been reported in various clinical phenotypes, such as syndromic/nonsyndromic and early/late onset. Among these, the most commonly reported causative genes are VEGFR3 [6, 7], causative for Milroy disease, and FOXC2 [8, 19-21], causative for lymphedema-distichiasis syndrome.

Among the 27 primary lymphedema patients included in this study, the family of only one patient was genetically confirmed to have a likely pathogenic variant of $C E L S R 1$, which has been previously reported as causative for nonsyndromic hereditary lymphedema. In 2016, Gonzalez-Galay et al. [22] reported a family with symptomatic hereditary lymphedema across three generations based on a proband with a mutation in CELSR1. In 2019, Erickson et al. [23] reported a family with CELSR1 haploinsufficiency in which lymphedema affected only the female members, and the patients presented with lymphangiectasia, valve dysfunction, and thoracic duct reflux. In 2021, Xia et al. [24] reported that a CELSR1 deletion could be associated with lymphatic dysplasia in a lymphedema patient with 22q13.3 deletion syndrome.

Morphological diagnosis of edema, functional imaging including lymphoscintigraphy and ICG lymphangiogram, and in particular, the absence of secondary etiologies such as cancer-related treatment, parasitic infections $[25,26]$ (e.g., filiarisis), and trauma are important for diagnosis of primary lymphedema. In the case of primary lymphedema of unknown etiology, there is very little information on disease progression, severity, and treatment strategies. However, as in the case presented in this study, early genetic detection in patients with nonsyndromic primary lymphedema enables accurate diagnosis of lymphedema and prompt intervention, such as rehabilitation and surgery. Additional case reports and genotype-phenotype information are needed to develop a more fundamental approach to primary lymphedema, which is still lacking.

The main limitation of the present study is the small sample size. The study cohort was limited to patients with primary lymphedema who visited a single institution for a certain period. However, given that primary lymphedema is an uncommon disease, it is difficult to perform studies on a large cohort. Studies involving not only Koreans but also other ethnicities are needed to gain accurate and detailed information about primary lymphedema. Additionally, the detection rate of mutations was not very high in our study, although some VUSs need to be evaluated further. Sufficient information is not available on causative genetic variants for primary lymphedema, warranting additional studies investigating the involvement of unknown candidate genes. 
In conclusion, this study served as an opportunity to highlight the heterogenic nature of primary lymphedema and bridge the knowledge gap for clinical information about primary lymphedema through clinical and genetic profiling of patients. In addition, we confirmed the diagnostic utility of WES in primary lymphedema. We also reported the possibility that a mutation in CELSR1, which to the best of our knowledge has not been reported before in this population, is a cause of nonsyndromic primary lymphedema in Koreans and East Asians. These results confirm the importance of genetic testing for primary lymphedema and highlight the need for further investigation of WES as an important tool for diagnosis of primary lymphedema, which can facilitate early medical intervention.

\section{Methods}

\section{Patients}

Primary lymphedema patients who visited Hereditary Lymphedema Clinic, Seoul National University Bundang Hospital (Seongnam, South Korea) between February 2020 and October 2021 were included in the present study. Primary lymphedema was diagnosed on the basis of symptoms, physical examination, patient history, and imaging studies. All patients underwent clinical examination by a rehabilitation specialist (EJY) and a lymphedema microsurgeon (YJM). Written informed consent was obtained from all patients. This study was performed in accordance with the Declaration of Helsinki (2013) and was approved by the Institutional Review Board of Seoul National University Bundang Hospital (B-2112-724103).

\section{Clinical profiling}

\section{Clinical diagnosis of lymphedema}

Clinical diagnosis of lymphedema was made based on the location and severity of the lymphedema. Lymphedema staging was performed based on the ISL staging criteria [27]. If the stage of each part of the involved limb was different, the staging was based on the site with the most severe lymphedema.

Medical history and onset and duration of lymphedema were recorded for each patient. The types of treatment that the patient had undergone previously were classified. In addition, the possibility of syndromic appearance other than lymphatic malformation was investigated to rule out other anomalies and diseases. Finally, patient family history was investigated, and patients with family members or close relatives with symptomatic lymphedema were noted.

\section{Volume estimation}

Preoperative limb circumferences were measured using tape measures. For the arms, measurements were made $10 \mathrm{~cm}$ above, at, $10 \mathrm{~cm}$ below, and $20 \mathrm{~cm}$ below the elbow. For the legs, measurements were made $20 \mathrm{~cm}$ above, $10 \mathrm{~cm}$ above, at, $10 \mathrm{~cm}$ below, and $20 \mathrm{~cm}$ below the knee. The volumes of the limbs were estimated using the formula of a truncated cone. 


\section{Imaging analysis}

Radionuclide lymphoscintigraphy and ICG lymphangiography were performed in all patients, and the diagnosis and lymphatic transport patterns of the patients were classified based on these images. Arm/leg dermal backflow grade $[28,29]$ and lymphoscintigraphy grade (Table 2) were measured through ICG lymphangiography using previously published methods and compared with ISL clinical stages of the patients.

\section{WES}

DNA was extracted from whole blood samples collected from the 27 patients. WES was performed on DNA samples enriched using the SureSelectXT Human all Exon 50 Mb kit (Agilent, Santa Clara, CA, USA) on the Illumina HiSeq sequencing system (Illumina, Inc., San Diego, CA, USA) with 100-bp paired end reads. Sequencing alignment and variant calling were performed using NextGENe software (SoftGenetics, State College, PA, USA).

\section{Variant filtering and interpretation of clinical significance}

Variants were screened for the following 60 lymphedema-related genes: ACVRL 1, AKT1, ARAF, ARHGAP31, BRAF, CBL, CCBE1, CCM2, CELSR1, CTNNB1, DCHS1, ELMO2, ENG, EPHB4, FAT4, FGFR1, FLT4, FOXC2, GATA2, GDF2, GJC2, GLMN, GNA11, GNA14, GNAQ, HGF, HRAS, IDH1, IDH2, KIF11, KRAS, KRIT1, MAP2K1, MAP2K2, MAP3K1, MAP3K3, MAPK1, MAPK14, MAPK3, MET, MTOR, NRAS, PDCD10, PDGFRB, PIEZ01, PIK3CA, PTEN, PTPN11, PTPN14, RAF1, RASA1, RIT1, SHOC2, SMAD4, SOS1, SOX18, $S T A M B P, T E K, T P 53$, and VEGFC. Exonic variants including nonsynonymous variants and intronic variants within $10 \mathrm{bp}$ from the exonic region were included in the analysis. Classification of each retained variant was performed according to the American College of Medical Genetics and Genomics and the Association for Molecular Pathology guidelines [30].

\section{Declarations}

\section{Acknowledgments}

This work was supported by the National Supporting Program for Genetic Diagnosis of Rare Diseases of the Korea Centers for Disease Control \& Prevention. We express our sincere gratitude to the patients who participated in this study.

\section{Author Contributions}

SHS performed data interpretation, statistical analysis, and wrote the manuscript. SJL and JKP collected the results. EJY recruited the patients and performed the physical examination of patients. BK, JSL, MJK, SSP, and MWS analyzed the genetic profile. YJM performed data interpretation, statistical analysis, and wrote the manuscript. All authors revised, reviewed, and approved the final manuscript. 


\section{Competing Interests Statement}

The authors declare no potential conflicts of interest.

\section{Data Availability}

The data that support the findings of this study are available from Seoul National University Bundang Hospital, but restrictions apply to the availability of these data, which were used under license for the current study, and so are not publicly available. Data are however available from the authors upon reasonable request and with permission of the corresponding author and the Institutional Review Board of Seoul National University Bundang Hospital.

\section{References}

1. Connell, F., Brice, G. \& Mortimer P. Phenotypic characterization of primary lymphedema. Ann. N. Y. Acad. Sci. 1131, 140-146 (2008).

2. Sleigh, B.C. \& Manna, B. Lymphedema. (StatPearls Publishing, 2022).

3. Sudduth, C.L. \& Greene, A.K. Primary lymphedema: update on genetic basis and management. Adv. Wound Care 10.1089/wound.2020.1338 (2021).

4. Rockson, S.G. \& Rivera, K.K. Estimating the population burden of lymphedema. Ann. N. Y. Acad. Sci. $1131,147-154$ (2008).

5. Deng, J., et al. Self-care status, symptom burden, and reported infections in individuals with lowerextremity primary lymphedema. J. Nurs. Scholarsh. 47, 126-134 (2015).

6. Brice, G., et al. Milroy disease and the VEGFR-3 mutation phenotype. J. Med. Genet. 42, 98-102 (2005).

7. Evans, A.L., et al. Identification of eight novel VEGFR-3 mutations in families with primary congenital lymphoedema. J. Med. Genet. 40, 697-703 (2003).

8. Fang, J., et al. Mutations in FOXC2 (MFH-1), a forkhead family transcription factor, are responsible for the hereditary lymphedema-distichiasis syndrome. Am. J. Hum. Genet. 67, 1382-1388 (2000).

9. Kazenwadel, J., et al. Loss-of-function germline GATA2 mutations in patients with MDS/AML or MonoMAC syndrome and primary lymphedema reveal a key role for GATA2 in the lymphatic vasculature. Blood 119, 1283-1291 (2012).

10. Grünewald, S., Matthijs, G. \& Jaeken, J. Congenital disorders of glycosylation: a review. Pediatr. Res. 52, 618-624 (2002).

11. Schook, C.C., et al. Primary lymphedema: clinical features and management in 138 pediatric patients. Plast. Reconstr. Surg. 127, 2419-2431 (2011).

12. Fastré, E., et al. Splice-site mutations in VEGFC cause loss of function and Nonne-Milroy-like primary lymphedema. Clin Genet. 94, 179-181 (2018). 
13. Kitsiou-Tzeli, S., et al. Milroy's primary congenital lymphedema in a male infant and review of the literature. In Vivo 24, 309-314 (2010).

14. Witte, M.H., et al. Phenotypic and genotypic heterogeneity in familial Milroy lymphedema. Lymphology 31, 145-155 (1998).

15. Milroy, W.F. Chronic hereditary edema: Milroy's disease. J. Am. Med. Assoc. 91, 1172-1175 (1928).

16. Milroy, W.F. An Undescribed Variety of Hereditary Oedema (Appleton, 1892).

17. Lee, B.B., et al. Diagnosis and treatment of primary lymphedema. Consensus document of the International Union of Phlebology (IUP)-2013. Int Angiol. 32, 541-574 (2013).

18. Hadjis, N.S., et al. The role of CT in the diagnosis of primary lymphedema of the lower limb. AJR Am. J. Roentgenol. 144, 361-364 (1985).

19. Dagenais, S.L., et al. Foxc2 is expressed in developing lymphatic vessels and other tissues associated with lymphedema-distichiasis syndrome. Gene Expr. Patterns 4, 611-619 (2004).

20. Kriederman, B.M., et al. FOXC2 haploinsufficient mice are a model for human autosomal dominant lymphedema-distichiasis syndrome. Hum. Mol. Genet. 12, 1179-1185 (2003).

21. Finegold, D.N., et al. Truncating mutations in FOXC2 cause multiple lymphedema syndromes. Hum. Mol. Genet. 10, 1185-1189 (2001).

22. Gonzalez-Garay, M.L., et al. A novel mutation in CELSR1 is associated with hereditary lymphedema. Vasc. Cell 8, 1 (2016).

23. Erickson, R.P., et al. Sex-limited penetrance of lymphedema to females with CELSR1 haploinsufficiency: a second family. Clin. Genet. 96, 478-482 (2019).

24. Xia, S., et al. Lymphedema complicated by protein-losing enteropathy with a 22q13. 3 deletion and the potential role of CELSR1: a case report. Medicine 100, e26307 (2021).

25. Cantey, P.T., et al. Increasing compliance with mass drug administration programs for lymphatic filariasis in India through education and lymphedema management programs. PLoS Negl. Trop. Dis. 4, e728 (2010).

26. Shenoy, R.K. Clinical and pathological aspects of filarial lymphedema and its management. Korean J Parasitol. 46, 119-125 (2008).

27. Executive Committee. The diagnosis and treatment of peripheral lymphedema: 2016 consensus document of the International Society of Lymphology. Lymphology 49, 170-184 (2016).

28. Yamamoto, T., et al. Indocyanine green-enhanced lymphography for upper extremity lymphedema: a novel severity staging system using dermal backflow patterns. Plast. Reconstr. Surg. 128, 941-947 (2011).

29. Yamamoto, T., et al. The earliest finding of indocyanine green lymphography in asymptomatic limbs of lower extremity lymphedema patients secondary to cancer treatment: the modified dermal backflow stage and concept of subclinical lymphedema. Plast. Reconstr. Surg. 128, 314e-321e (2011). 
30. Richards, S., et al. Standards and guidelines for the interpretation of sequence variants: a joint consensus recommendation of the American College of Medical Genetics and Genomics and the Association for Molecular Pathology. Genet Med. 17, 405-424 (2015).

\section{Tables}

Table 1,2 is available in the Supplemental Files section.

\section{Figures}




\section{ONSET OF LYMPHEDEMA}

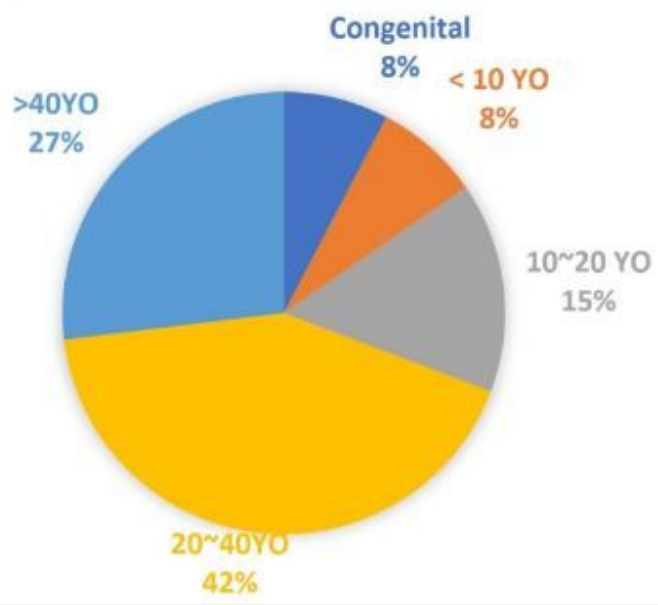

\section{LOCATION OF LYMPHEDEMA}

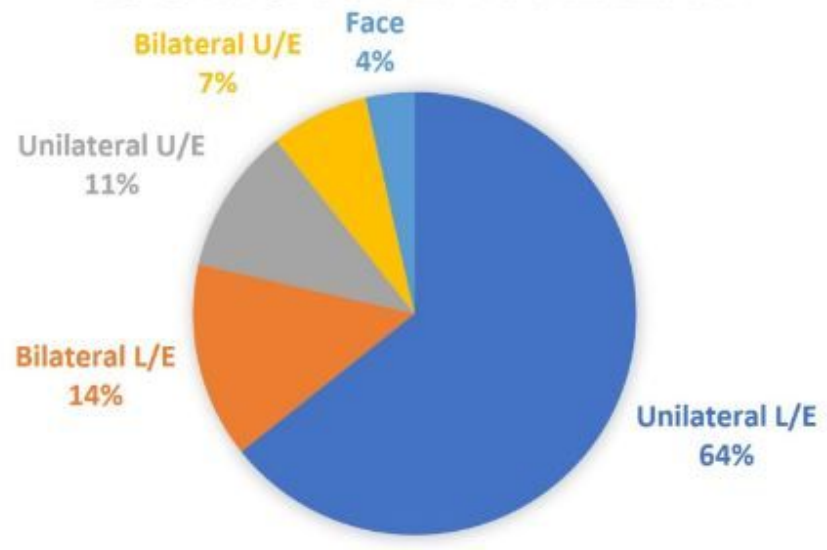

ISL STAGE OF LYMPHEDEMA

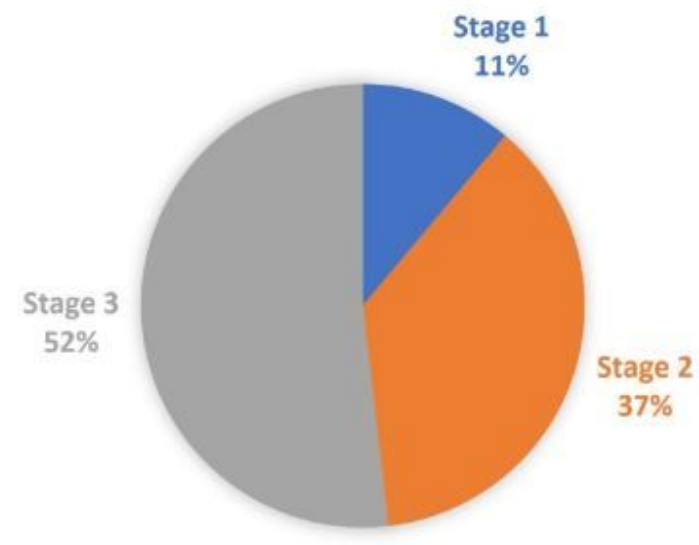

Figure 1

Onset (above), location (middle), and stages (below) of primary lymphedema in the patient cohort. 


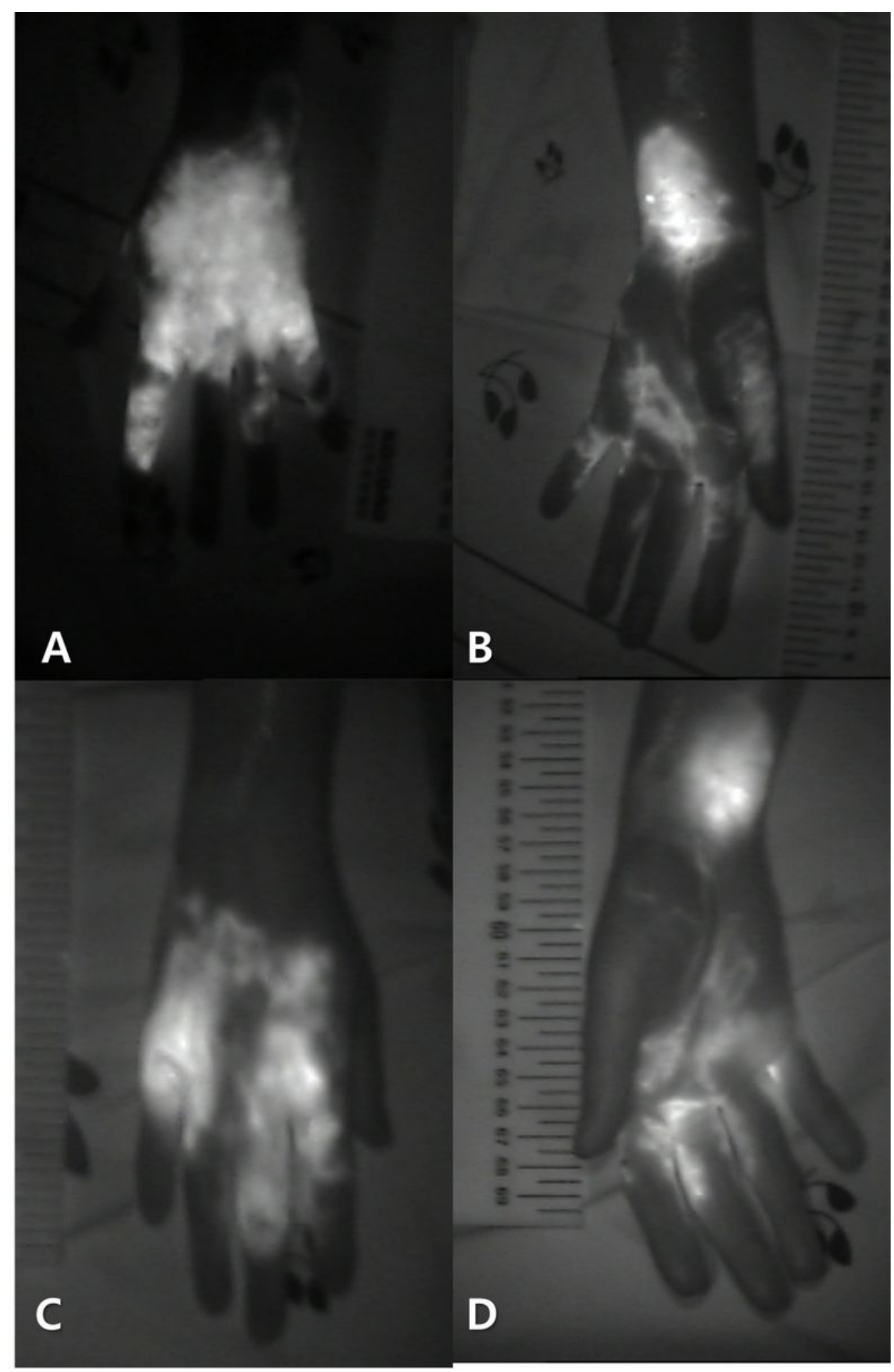

\section{Figure 2}

ICG lymphangiography of the patient presented as case 1. (a) Left hand dorsal side, (b) left hand palm side, (c) right hand dorsal side, (d) right hand palm side. Dermal backflow and lymphatic retention were observed on both sides, but the left side showed a more severe pattern. 


\section{Figure 3}

Lymphoscintigraphy of the patient presented as case 1. Arm images taken (a) 5 min, (b) 30 min, and (c) 60 min after radionuclide injection. Chest images taken (d) 5 min, (e) $30 \mathrm{~min}$, and (f) $60 \mathrm{~min}$ after radionuclide injection.

\section{Figure 4}

Microscopic operative photographs taken during lymphovenous anastomosis. (a) Lymphatic vessel (above) and vein (below). (b) Fluorescence mode shows good lymphatic flow in lymphatic vessel. (c) After lymphovenous anastomosis. (d) Good lymphatic flow confirmed through the bypass.

\section{Supplementary Files}

This is a list of supplementary files associated with this preprint. Click to download.

- Table1.xlsx

- Table2.xlsx 